\title{
INERTIAL AUTOMORPHISMS OF A CLASS OF WILDLY RAMIFIED $v$-RINGS
}

\author{
BY \\ NICKOLAS HEEREMA( $\left.{ }^{1}\right)$
}

I. Introduction. Let $R_{e}$ be a ramified $v$-ring with ramification $e$. That is, $R_{e}$ is a complete, discrete, rank one, valuation ring having characteristic zero with residue field $k$ of characteristic $p(p \neq 0,2)$ and $p R_{e}$ is the $e$ th power of the maximal ideal $M$ of $R_{e}$. Let (Ss represent the group of automorphisms of $R_{e}, \varepsilon$ being the identity map. Then, for $i>0, \mathscr{S}_{i}=\left\{\alpha \mid \alpha \in \mathscr{S F} ; \alpha=\varepsilon, \bmod M^{i}\right\}$ and $\mathfrak{S}_{i}=\left\{\alpha \mid \alpha \in \mathscr{S F}_{i}\right.$, $\alpha(m)-m \in M^{i+1}$ for $\left.m \in M\right\}$. The ramification groups $\mathfrak{S}_{i}$ and $\mathfrak{S}_{i}$ are invariant in (5). The object of this paper is to evaluate the factor groups of the series (1) of

$$
\mathfrak{S S}_{i} \supseteq \mathfrak{S}_{1} \supseteq \mathfrak{S H}_{2} \supseteq \mathfrak{S}_{2} \supseteq \cdots
$$

ramification groups in that case in which $e=p$. A second objective is the determination of those automorphisms in $\mathbb{S}_{1}$ which are derivation automorphisms (see below).

Neggers has shown [3, Theorem 6] that for any $e$ and $i \geqq(e+p) /(p-1)$, $\mathfrak{S}_{i} / \mathscr{S}_{i+1}$ is isomorphic to $\mathscr{D}\left(R_{e}\right) / \pi \mathscr{D}\left(R_{e}\right)$ where $\mathscr{D}\left(R_{e}\right)$ is the additive group of derivations on the ring $R_{e}$ and $\pi \mathscr{D}\left(R_{e}\right)=\left\{\pi d \mid d \in \mathscr{D}\left(R_{e}\right)\right\}$ where $\pi$ is a prime element in $R_{e}$. In addition he proved that $\mathfrak{S}_{i} / \mathscr{S}_{i+1}$ is isomorphic to the additive group of those derivations on $k$ which lift to $R_{e}$ where again $i \geqq(e+p) /(p-1)$. The map used by Neggers to evaluate $\mathscr{G}_{i} / \mathscr{S}_{i+1}$ also shows that if $i \geqq(e+p) /(p-1)$, then $\mathfrak{S}_{i} / \mathscr{S}_{i}$ is isomorphic to $\mathscr{D}\left(R_{e}\right) / \mathscr{D}^{*}\left(R_{e}\right)$ where $\mathscr{D}^{*}\left(R_{e}\right)=\left\{d \mid d \in \mathscr{D}\left(R_{e}\right), d(\pi) \in \pi R_{e}\right\}$ [3, proof of Theorem 6]. The principal tool of this investigation is the convergent higher derivation [2]. Let $D=\left\{D_{i}\right\}_{i=1}^{\infty}$ be a higher derivation on $R_{e}\left(D_{i}\left(R_{e}\right) \subset R_{e}\right.$ for $i>0)$. $D$ is convergent if, for $a \in R_{e}, \sum D_{i}(a)$ is a convergent series in the $\pi$-adic topology. If $D$ converges the map $\alpha_{D}: a \rightarrow \sum_{i=0}^{\infty} D_{i}(a)\left(D_{0}(a)=a\right)$ is an inertial automorphism (see Theorem B). The group $\mathfrak{S S}_{D}$ of all derivation automorphism $\alpha_{D}$ is an invariant subgroup of (B).

Throughout this paper $R$ will denote a $v$-ring in $R_{e}$ such that $\left[R_{e}: R\right]=e$, and $R$ is unramified. Thus $R$ has the same residue field $k$ as $R_{e}$. For $a$ in $R_{e}, \bar{a}$ will denote the image of $a$ under the natural map of $R_{e}$ onto $k, \pi$ always represents a prime element in $R_{e}$. We have

$$
\pi^{e}+p u=0, \quad \bar{u} \neq 0 .
$$

If $e=p$ and $\bar{u} \in k^{p}$ then $\pi$ can be chosen so that

$$
\pi^{p}+p\left(1+\pi^{t} v\right)=0, \quad t>0, \quad \bar{v} \neq 0,
$$

or $\pi^{p}+p=0$. We note that the conditions

$$
\bar{u} \notin k^{p} ; t=p \text { and } \quad \bar{v} \notin k^{p} \text {; as well as } \bar{v} \text { if } 1 \leqq t<p
$$

Presented to the Society, September 1, 1967; received by the editors February 16, 1967.

(1) This research supported by NSF GP-4007. 
are all independent of the choice of $\pi$, assuming (3) to be satisfied for all but the first listed. Throughout this paper the symbols $u$ and $v$ will represent the quantities given in (2) and (3). The considerations (4) determine, to a large extent, the structure of the groups (1) as is seen in the following two theorems which summarize the results of this study. $\mathfrak{S}\left(R_{p} ; R\right)$ is the group of automorphism of $R_{p}$ over $R$.

THEOREM 1. Every inertial automorphism on $R_{p}$ is a derivation automorphism, i.e. $\mathfrak{B S}_{1}=\mathfrak{S}_{D}$, unless $\bar{u} \in k^{p}$ and $t=p-1$, in which case the following are equivalent.

(a) $\bar{v}$ is $a(p-1)$ th root in $k$.

(b) $R_{p}$ is Galois over $R$.

(c) $\mathbb{S}_{2} \neq \mathfrak{S}_{2}$.

(d) $\mathfrak{S}_{2} \mid \mathfrak{S}_{2}$ is the group of order $p$.

If $R_{p}$ is Galois over $R$ then $\mathfrak{S}\left(R_{p} ; R\right) \subset \mathfrak{S}_{D}$ if and only if $\bar{v} \notin k^{p}$. In any case, $\mathbb{G S}_{1}=\mathbb{S S}_{D} \cdot \mathbb{S S}\left(R_{p} ; R\right)$.

THEOREM 2. If $\bar{u} \notin k^{p}$ then for $i \geqq 1, \mathfrak{S}_{\mathfrak{i}} / \mathscr{S}_{i+1}$ is isomorphic to the subgroup $\overline{\mathscr{D}}$ of those $\delta \in \mathscr{D}(k)$ which lift to $R_{p}$. Also, $\overline{\mathscr{D}}=\{\delta \mid \delta \in \mathscr{D}(k), \delta(\bar{u})=0\}$. In this case $\mathfrak{S S}_{i} / \mathfrak{S}_{i}$ is isomorphic to $k^{+}$, the additive group of $k$.

If $\bar{u} \in k^{p}$, then for $i \geqq 1, \mathfrak{S}_{i} / \mathscr{S}_{i+1}$ is isomorphic to $\mathscr{D}(k)$ unless $t=p$ and $i=1$. If $t=p, \mathscr{S}_{1} / \mathscr{S}_{2}$ is isomorphic to the subgroup of those $\delta \in \mathscr{D}(k)$ such that $\delta(\bar{v})=0$. Also, $\mathbb{B S}_{i}=\mathfrak{S}_{i}, i \geqq 1$, unless $t=p-1, i=2$ and one of the four equivalent conditions of Theorem 1 holds.

By Neggers' results referred to above [3, proof of Theorem 6] we have

CoRollaRY. $\mathscr{D}\left(R_{p}\right) / \mathscr{D}^{*}\left(R_{p}\right)$ is isomorphic to $k^{+}$if $\bar{u} \notin k^{p}$ and is trivial if $\bar{u} \in k^{p}$.

II. Proofs. For $S$ a subring of $R_{e}$, the symbols $\mathscr{H}\left(S, R_{e}\right), \mathscr{H}_{c}\left(S, R_{e}\right)$ and $\mathscr{H}_{u}\left(S, R_{e}\right)$ will stand for the set of all higher derivations, all convergent higher derivations, and all uniformly convergent higher derivations with domain $S$ and range $R_{e}$. We quote the following two theorems which will be used repeatedly. Theorem A provides the necessary freedom in the construction of $D \in \mathscr{H}_{u}\left(R_{e}, R_{e}\right)$. Theorem B implies that if $D \in \mathscr{H}_{c}\left(R_{e}, R_{e}\right)$, then $\alpha_{D}$ is indeed an inertial automorphism.

THEOREM A [2, Theorem 4]. Let $\overline{\mathscr{S}}$ be a p-basis for $k$ and let $\mathscr{S} \subset R$ be a set of representatives of the elements of $\overline{\mathscr{S}}$. If $I$ is the set of positive integers and $f$ is a mapping from $\mathscr{S} \times I$ into $R_{e}$ then there is one and only one $D \in \mathscr{H}\left(R, R_{e}\right)$ such that $D_{i}(s)=f(s, i)$ for all $s \in \mathscr{S}$ and $i \in I$. Moreover, $D$ converges (uniformly) if and only if $D$ converges (uniformly) on $\mathscr{S}$.

THEOREM B [2, Lemmas 1 and 5]. If $D$ is in $\mathscr{H}_{c}\left(R_{e}, R_{e}\right)$ then $D_{i}\left(R_{e}\right) \subset \pi R_{e}$ and $D_{i}\left(\pi R_{e}\right) \subset \pi^{2} R_{e}$ for $i>0$.

Theorems 1 and 2 will be proved by means of a series of lemmas. 
LEMMA 1. If $\mathscr{S}$ is a set of representatives in $R$ of a p-basis $\overline{\mathscr{S}}$ for $k$ and $D$ in $\mathscr{H}\left(R, R_{e}\right)$ is such that $D_{j}(\mathscr{S}) \subset \pi^{t}, R_{e} \subset \pi R_{e}, j \geqq 1$, then $D_{i}(R) \subset \pi^{q_{i}} R_{e}$ where

$$
q_{i}=\min _{j_{1}+\cdots+j_{i}=i: t_{0}=0}\left(t_{j_{1}}+\cdots+t_{j_{i}}\right), \quad i \geqq 1 .
$$

Proof. For a given $i$ we choose $n$ sufficiently large so that $D_{j}\left(R^{p^{n}}\right) \subset \pi^{q_{i}} R_{e}$ for $j=1, \ldots, i\left[2\right.$, Lemma 6] where $R^{p^{n}}$ is the subring of $R$ generated by the $p^{n}$ th powers of elements in $R$. Since every element in $k$ has a representative in $R^{p^{n}}[\mathscr{S}$, it follows that $R=R^{p^{n}}[\mathscr{S}]+p^{q_{i}} R$. If $b=a s_{1}, \ldots, s_{r}$ where $a \in R^{p^{n}}$ and $s_{1}, \ldots$, $s_{r} \in \mathscr{S}$ then

$$
D_{\imath}(b)=\sum_{i_{0}+\cdots+i_{r}=i} D_{i_{0}}(a) D_{i_{1}}\left(s_{1}\right), \ldots, D_{r}\left(s_{r}\right)
$$

is seen to be in $\pi^{q_{l}} R$ and hence, since $D_{j}\left(p^{q_{i}}\right)=0$ for all $j, D_{i}(R) \subset \pi^{q_{l}} R_{e}$.

Let $\alpha$, an automorphism of $R_{e}$, be in $\mathfrak{S}_{i}, i \geqq 1$. Then $\alpha(a)=a+\pi^{i} \alpha^{*}(a)$ and the mapping $\alpha^{*}$ induces a derivation $\delta_{\alpha}$ on $k$. The mapping

$$
\phi_{i}: \alpha \rightarrow \delta_{\alpha}
$$

is a homomorphism of $\mathscr{S}_{i}$ into $\mathscr{D}(k)$ with kernel $\mathscr{S}_{i+1}$.

LEMMA 2. If an automorphism $\alpha$ of $R_{p}$ is in $\mathfrak{H}_{i}$, then $\delta_{\alpha}(\bar{u})=0$. If $\pi^{p}+p\left(1+\pi^{p} v\right)=0$, then $\delta_{\alpha}(\bar{v})=0$ for $\alpha$ in $\mathfrak{S}_{1}$.

Proof. Since $\alpha(\pi)-\pi \in \pi^{i+1} R_{p}$ it follows that $\alpha\left(\pi^{p}\right)-\pi^{p} \in \pi^{p+i+1} R_{p}$. Thus by (2) $\alpha(u)-u$ is in $\pi^{i+1} R_{p}$ or $\alpha^{*}(u) \in \pi R_{p}$ which implies $\delta_{\alpha}(\bar{u})=0$. In the remaining case let $\alpha(\pi)=\pi+\pi^{2} b$. Then $\alpha\left(\pi^{p}\right)-\pi^{p} \equiv \pi^{2 p} b^{p}, \bmod \pi^{2 p+1} R_{p}$, and $\alpha\left(p\left(1+\pi^{p} v\right)\right)-p\left(1+\pi^{p} v\right)$ $\equiv p \pi^{p+1} \alpha^{*}(v), \bmod \pi^{2 p+2} R_{p}$. Hence $b \in \pi R_{p}$ and it follows that $\alpha^{*}(v) \in \pi R_{p}$ or $\delta_{\alpha}(\bar{v})=0$.

Given $D$ and $H$ in $\mathscr{H}\left(R_{e}, R_{e}\right), D \circ H$ in $\mathscr{H}\left(R_{e}, R_{e}\right)$ is given by

$$
(D \circ H)_{i}=\sum_{j=0}^{i} D_{j} H_{i-j}
$$

$\mathscr{H}\left(R_{e}, R_{e}\right)$ is a group with respect to this composition and $\mathscr{H}_{c}\left(R_{e}, R_{\varepsilon}\right), \mathscr{H}_{u}\left(R_{e}, R_{e}\right)$ are subgroups [2, Theorems 1, 2]. Moreover, one can verify directly that, for $D$ and $H$ in $\mathscr{H}_{c}\left(R_{e}, R_{e}\right), \alpha_{D \circ H}=\alpha_{D} \alpha_{H}$.

Lemma 3. Let $\left\{D^{(n)}\right\}_{n=1}^{\infty}$ where $D^{(n)} \in \mathscr{H}_{c}\left(R_{e}, R_{e}\right)$ be such that $D_{i}^{(n)}\left(R_{e}\right) \subset \pi^{s_{n}} R_{e}$, $i \geqq 1, n \geqq 1$, and $\lim _{n} s_{n}=\infty$. Let $\alpha_{n}=\alpha_{D^{(1)}}, \ldots, \alpha_{D^{(n)}}$ and $\bar{D}^{(n)}=D^{(1)} \circ \ldots \circ D^{(n)}$. Then $\lim _{n} \alpha_{n}(a)$ and $\lim _{n} \bar{D}_{i}^{(n)}(a)$ exist for all $i>0$ and $a \in R_{e}$. Moreover, $\alpha: a \rightarrow$ $\lim _{n} \alpha_{n}(a)$ is an automorphism, $D=\left\{D_{i}\right\}$ is in $\mathscr{H}_{c}\left(R_{e}, R_{e}\right)$ where $D_{i}(a)=\lim _{n} \bar{D}_{i}^{(n)}(a)$ and $\alpha=\alpha_{D}$. If $D^{(n)} \in \mathscr{H}_{u}\left(R_{e}, R_{e}\right) n \geqq 1$, then $D \in \mathscr{H}_{u}\left(R_{e}, R_{e}\right)$.

Proof. By definition of product in $\mathscr{H}_{c}\left(R_{e}, R_{e}\right)$ we have

$$
D_{n}^{(m+1)}(a)-D_{n}^{(m)}(a)=\sum_{i_{1}+\cdots+i_{m+1}=n ; i_{m+1} \neq 0} D_{i_{1}}^{(1)} \cdots D_{i_{m+1}}^{(m+1)}(a),
$$


the right side of which is in $\pi^{s_{m+1}} R_{e}$ since for $D \in \mathscr{H}_{c}\left(R_{e}, R_{e}\right), D_{i}\left(\pi^{n} R_{e}\right) \subset \pi^{n} R_{e}$ for all $i$ and $n$ by Theorem B. Thus, $\alpha_{m+1}(a)-\alpha_{m}(a)=\sum_{i=0}^{\infty} \bar{D}_{i}^{(m+1)}(a)-\sum_{i=0}^{\infty} \bar{D}_{i}^{(m)}(a)$ $\in \pi^{s_{m+1}} R_{e}$. The rest of the lemma follows directly.

Since $R_{p}$ is totally ramified over $R$ and $\left[R_{p}: R\right]=p$ then $R_{p}=R[\pi]$ and the minimal polynomial $f(x)$ of $\pi$ over $R$ is an Eisenstein polynomial, that is,

$$
f(x)=x^{p}+p a_{p-1} x^{p-1}+\cdots+p a_{1} x+p a_{0}
$$

and $a_{0}$ is a unit. Clearly, $\bar{a}_{0}=\bar{u}$ (see (2)). Also, if $\bar{u} \in k^{p}$ then $a_{0}=b^{p}+p c$ where $b$ and $c$ are in $R$. By replacing $\pi$ with $b^{-1} \pi$ we can assume that

$$
a_{0}=1+p b_{0} \text {. }
$$

We note next that every $D \in \mathscr{H}\left(R, R_{e}\right)$ extends uniquely to a higher derivation $D$ of the quotient field of $R_{e}$. Also, $D\left(R_{e}\right) \subset R_{e}$ if and only if $D(\pi) \in R_{e}$. If $D$ converges on $R, D$ will converge on $R_{e}$ if and only if $D$ converges at $\pi$ [2, Lemma 3].

Let $(r, s)$ denote an ordered set of $r$ nonnegative integers whose sum is $s$ and let $|(r, s)|$ represent the largest integer in $(r, s)$. We let $\sum_{(q, s)} D\left(a_{1}, \ldots, a_{q}\right)$ denote the sum of all products $D_{i_{1}}\left(a_{1}\right) D_{i_{2}}\left(a_{2}\right) \cdots D_{i_{q}}\left(a_{q}\right)$ such that $i_{1}+\cdots+i_{q}=s$ and $i_{j} \geqq 0$. Also, $f^{\prime}(x)$ and $f^{D_{i}}(x)$ represent respectively the ordinary derivative of $f$ and the polynomial obtained by replacing each coefficient in $f$ with its image under $D_{i}$. With these conventions it is useful to write the expression for $D_{i}(\pi)$ derived from $D_{i}(f(\pi))=0$ as follows:

$$
f^{\prime}(\pi) D_{i}(\pi)=f^{D_{l}}(\pi)+\sum_{(p, i): i(p, i) \mid<i} D(\pi, \ldots, \pi)
$$

$$
+\sum_{j=0}^{p-1} p \sum_{(j+1, i) ;(j+1, i) \mid<i} D\left(a_{j}, \pi, \ldots, \pi\right) .
$$

Let $v$ represent the exponential valuation on $R_{p}$. Note that $p \leqq v\left(f^{\prime}(\pi)\right) \leqq 2 p-1$.

LeMma 4. A given $\delta \in \mathscr{D}(k)$ lifts to $d \in \mathscr{D}\left(R_{p}\right)$ if and only if $\delta(\bar{u})=0$.

Proof. A derivation $d \in \mathscr{D}\left(R_{p}\right)$ induces a derivation on $k$ under the natural map of $R_{p}$ onto $k$ only if $d(\pi) \subset \pi R_{p}$. But $d(f(\pi))=0$ means $d(\pi)=-f^{d}(\pi) / f^{\prime}(\pi)$. Thus $f^{d}(\pi) \in \pi^{p+1} R_{p}$ which means $d\left(a_{0}\right) \in \pi R_{p}$ and, hence, if $d$ induces $\delta$ on $k$, $\delta\left(\bar{a}_{0}\right)=\delta(\bar{u})=0$. Conversely, every $\delta \in \mathscr{D}(k)$ lifts to $d^{\prime}$ on $R$ [1, Theorem 1]. If $\delta(\bar{u})=0, d^{\prime}\left(a_{0}\right) \in p R$ which means that $f^{d^{\prime}}(\pi) / f^{\prime}(\pi) \in \pi R_{p}$. Thus the extension $d$, of $d^{\prime}$, to $R_{p}$ is in $\mathscr{D}\left(R_{p}\right)$ and induces $\delta$ since $d^{\prime}$ does.

Lemma 5. Let $D \in \mathscr{H}_{c}\left(R, R_{p}\right)$ where $R_{p}=R[\pi]$ and $f(x)=x^{p}+\sum_{i=0}^{p-1} p a_{i} x^{i}$ is the minimum function of $\pi$ over $R$. Let $q>2, n \geqq 1$, and $m>p(n-1)$ be integers such that, using the same symbol for the extension of $D$ to $R_{p}$,

$$
\begin{array}{ll}
D_{j}(\pi) \in \pi^{2} R_{p} & \text { if } j<n, \\
D_{j}(\pi) \in \pi^{q} R_{p} & \text { if } n \leqq j<m,
\end{array}
$$

and, if $j \geqq n$

$$
D_{j}\left(a_{s}\right) \in f^{\prime}(\pi) \pi^{q-p-s} .
$$


If $q=3$ then $(9,3)$ is assumed to hold only for $j \geqq m$. Under these assumptions $\sum D_{j}(\pi)$ converges and

$$
\sum_{j=n}^{\infty} D_{j}(\pi) \in \pi^{q} R_{p}
$$

The proof of this lemma consists of checking the valuation $v$ of the terms on the right side of (8). We show first that if $j \geqq m$,

$$
D_{j}(\pi) \in \pi^{q} R_{p} \text {. }
$$

Thus assuming (10) true for $j<r$ where $r \geqq m$ we consider $(8, \mathrm{r})$. By $(9,3) f^{D_{r}(\pi)}$ is in $f^{\prime}(\pi) \pi^{q} R_{p}$. The term $D_{i_{1}}(\pi) \cdots D_{i_{p}}(\pi)$ of $A_{r}=\sum_{(p, r):|(p, r)|<r} D(\pi, \ldots, \pi)$ is in $\pi^{p+q} R_{p}$ in view of the fact that at least one $i_{j}>n$ and another is different from zero. The above term appears in $A_{r}$ a multiple of $p$ times unless $i_{1}=i_{2}=\cdots=i_{p}=r / p$ in which case it is in $\pi^{p q} R_{p} \subset f^{\prime}(\pi) \pi^{q} R_{p}$, since $v\left(f^{\prime}(\pi)\right) \leqq 2 p-1$ and $p \neq 2$. Thus $A_{r} \in f^{\prime}(\pi) \pi^{q} R_{p}$. A similar argument shows $B_{r}$, the remaining term on the right side of $(8, \mathrm{r})$, to be in $f^{\prime}(\pi) p^{q} R_{p}$. Thus by $(8, \mathrm{r}) D_{r}(\pi) \in \pi^{q} R_{p}$.

Given $i \geqq 0$, we assume for some integer $s \geqq m$ that if $j>s$, then $D_{j}(\pi) \in \pi^{i+a} R_{p}$ and, for $h=0, \ldots, p-1$

$$
D_{j}\left(a_{h}\right) \in f^{\prime}(\pi) \pi^{i+q-p+1} R_{p}
$$

Let $s^{\prime}=p s$ and let $j>s^{\prime}$. Then $f^{D_{j}}(\pi) \in f^{\prime}(\pi) \pi^{i+q+1} R_{p}$ and, by an anasis like that above, $A_{j}$ and $B_{j}$ are seen to be in $f^{\prime}(\pi) \pi^{i+q+1} R_{p}$. Thus $D_{j}(\pi) \in \pi^{i+q+1} R_{p}$. Since $D$ converges on $R$, given $i \geqq 0$, there is an $s$ such that (11) holds for $j>s$. It follows that $\sum D_{j}(\pi)$ converges, and in view of $(10) \sum_{j=n}^{\infty} D_{j}(\pi) \in \pi^{q} R_{p}$.

Lemma 6. If $\alpha \in \mathfrak{S}_{\mathfrak{i}}, i \geqq 1$, then there is a $D \in \mathscr{H}_{u}\left(R_{p}, R_{p}\right)$ such that $\alpha^{-1} \alpha_{D} \in G_{i+1}$. Moreover, $\mathfrak{S}_{i} / \mathscr{S}_{i+1}$ is isomorphic to the subgroup of those $\delta$ in $\mathscr{D}(k)$ for which $\delta(\bar{u})=0$ with the following exception. If $\bar{u} \in k^{p}$ and, for suitable choice of $\pi$ we have $\pi^{p}=p\left(1+\pi^{p} v\right)$ then $\mathfrak{S}_{1} / \mathscr{S}_{2}$ is isomorphic to the subgroup of those

$$
\delta \in \mathscr{D}(k) \ni \delta(\bar{v})=0 .
$$

Proof. By Lemma 2 , it will be sufficient to find $D \in \mathscr{H}_{u}\left(R_{p}, R_{p}\right)$ such that $\phi_{i}\left(\alpha_{D}\right)$ (see (5)) is a given $\delta$ for which $\delta(\bar{u})=0$, or, in the exceptional case, $\delta(\bar{v})=0$. Let (6) be the minimum function of $\pi$ over $R$.

Case 1. $v\left(f^{\prime}(\pi)\right)<2 p-1, i>1$. Let $\delta$ be any derivation on $k$ for which $\delta(\bar{u})=$ $\delta\left(\bar{a}_{0}\right)=0$ and let $H=\left\{H_{j}\right\}$ be any higher derivation in $\mathscr{H}(R, R)$ satisfying the two conditions (a) $H_{1}$ induces $\delta$, (b) $H_{j}\left(a_{0}\right) \in p R, j=1, \ldots, p-1$. Specifically, every derivation on $k$ lifts to $R$ [1, Theorem 1] which fact makes $H_{1}$ available. Let $H_{j}=H_{1}^{j} / j$ ! for $j=2, \ldots, p-1$. By Theorem A, maps $H_{j}, j \geqq p$, can be defined so that $H=\left\{H_{j}\right\} \in \mathscr{H}(R, R)$. Let $D=\left\{D_{j}\right\}$ where

$$
D_{j}=\pi^{j i} H_{j} \text {. }
$$

Clearly, $D \in \mathscr{H}_{u}\left(R, R_{p}\right)$. We now show that

$$
D_{j}(\pi) \in \pi^{i+1} R_{p}, \quad j \geqq 1,
$$

$$
\sum D_{j}(\pi) \text { converges, }
$$


from which, with (12), it will follow that, using the same symbol for the extended higher derivation, $D \in \mathscr{H}_{u}\left(R_{p}, R_{p}\right), \alpha_{D} \in \mathfrak{H}_{i}$ and $\Phi_{i}\left(\alpha_{D}\right)=\delta$.

Let $v\left(f^{\prime}(\pi)\right)=p+r-1$. Thus $r$ is the least positive integer such that $a_{r}$ is a unit. Looking to the conditions of Lemma 5 we note that $f^{\prime}(\pi) \pi^{i+1-p-s} R_{p}=\pi^{i+r-s} R_{p}$. If $s \geqq r, D_{j}\left(a_{s}\right) \in \pi^{j i} R_{p} \subset \pi^{i+r-s} R_{p}$ for $j \geqq 1$. If $r>s>0, a_{s} \in p R$; hence, $H_{j}\left(a_{s}\right) \in p R$ and thus $D_{j}\left(a_{s}\right) \in \pi^{i j+p} R_{p} \subset \pi^{i+r-s} R_{p}$ for $j \geqq 1$. Finally, $D_{j}\left(a_{0}\right)=\pi^{i j} H_{j}\left(a_{0}\right)$. If $j<p$, $H_{j}\left(a_{0}\right) \in p R_{p}$ and $\pi^{i j} H_{j}\left(a_{0}\right) \in \pi^{i j+p} R_{p} \subset \pi^{i+r} R_{p}$. If $j \geqq p, i j \geqq i+r$. Hence, $D_{j}\left(a_{0}\right)$ $\in \pi^{i+r} R_{p}$ for $j \geqq 1$. Thus conditions $(9,1)$ to $(9,3)$ are satisfied with $q=i+1$ and $n=m=1$. Hence (13) and (14) hold.

Case 2. $v\left(f^{\prime}(\pi)\right)<2 p-2, i=1$. We define $D$ as in Case 1 and note by inspection of $(8, \mathrm{j})$ for $j=1, \ldots, p$ that $D_{1}(\pi) \in \pi^{2} R_{p}, D_{j}(\pi) \in \pi^{3} R_{p}$ for $j=2, \ldots, p$. Also, in this case, $f^{\prime}(\pi) \pi^{3-p-s} R_{p} \supset \pi^{p-s} R_{p} \supset \pi^{p} R_{p}$ and, by (12) $D_{j}\left(a_{\mathrm{s}}\right) \in \pi^{p} R_{p} \subset f^{\prime}(\pi) \pi^{3-p-s} R_{p}$ for $j \geqq p$ and $s=0, \ldots, p-1$. Thus conditions $(9,1)$ to $(9,3)$ are satisfied for $n=2$, $m=p+1$ and $q=3$. Hence $\sum D_{i}(\pi)$ converges and is in $\pi^{2} R_{p}$. Thus, $\alpha_{D} \in \mathfrak{S}_{1}$ and $\phi_{1}\left(\alpha_{D}\right)=\delta$.

Case 3. $v\left(f^{\prime}(\pi)\right)=2 p-2, i=1$. We consider a number of subcases. In each case $D$ is constructed by the method of Theorem A.

(3, i). $\bar{a}_{p-1} \notin k^{p}, \bar{a}_{0} \notin k^{p}, \bar{a}_{p-1}$ and $\bar{a}_{0} p$-independent. As before, we initiate the construction of $D \in \mathscr{H}_{u}\left(R, R_{p}\right)$ by letting $D_{j}=\pi^{j} H_{j}, j=1, \ldots, p-1$, where $\left\{H_{j}\right\}_{1}^{p-1}$ are chosen so that $H_{1}$, a derivation on $R_{p}$ induces a given $\delta \in \mathscr{D}(k)$ such that $\delta\left(\bar{a}_{0}\right)=0$ and $H_{j}\left(a_{0}\right) \in p R$ for $j=2, \ldots, p-1$. Let $\mathscr{S}$ be a set of representatives in $R$ of a $p$-basis $\overline{\mathscr{S}}$ of $k$. We may assume both $a_{0}$ and $a_{p-1}$ in $\mathscr{S}$. By inspection of $(8,1)$ to $(8, p-1)$ we have $D_{1}(\pi) \in \pi^{2} R_{p}$ and $D_{j}(\pi) \in \pi^{3} R_{p}, j=2, \ldots, p-1$. Considering $(8, \mathrm{p})$, each summand of $A_{p}$ and $B_{p}$ is in $\pi^{2 p+1} R_{p}$, except the term $\left[D_{1}(\pi)\right]^{p}$ which is in $\pi^{2 p} R_{p}$ but not in $\pi^{2 p+1} R_{p}$. Thus, we define $D_{p}$ by $D_{p}(s)=0$ for $s \in \mathscr{S}-\left\{a_{0}\right\}$ and $D_{p}\left(a_{0}\right)$ is so chosen that $f^{D_{p}}(\pi)+A_{p}+B_{p}$ is in $f^{\prime}(\pi) \pi^{3} R_{p}$. Thus, $D_{p}\left(a_{0}\right) \in \pi^{p} R_{p}$ and $D_{p}(\pi) \in \pi^{3} R_{p}$. For $j>p$ we let $D_{j}(s)=0$ for $s \in \mathscr{S}$. By Lemma $1 D_{j}\left(a_{s}\right) \in \pi^{p+1} R_{p}$ for $j>p$. It follows that conditions (9) of Lemma 5 are fulfilled for $n=2, m=p+1$ and $q=3$. Thus by Theorem A the extension of $D$ to $R_{p}$ converges uniformly $\alpha_{D} \in \mathfrak{S}_{1}$ and $\Phi_{1}\left(\alpha_{D}\right)=\delta$.

(3, ii) $\bar{a}_{p-1} \notin k^{p}, \bar{a}_{0} \notin k^{p}, \bar{a}_{p-1}$ and $\bar{a}_{0} p$-dependent. Let $H_{1} \in \mathscr{D}(R)$ induce $\delta \in \mathscr{D}_{k}$ where $\delta\left(\bar{a}_{0}\right)=0$ (Lemma 4 ) and let $\mathscr{S}$ be a set of representatives of a $p$-basis for $k$ which contains $a_{0} . H \in \mathscr{H}_{u}(R, R)$ is defined by the conditions $H_{j}(s)=0$ for $j>1$ and $s \in \mathscr{S}$. Let $D_{j}=\pi^{j} H_{j}$ for $j \geqq 1$. Now $H_{1}\left(a_{p-1}\right) \in p R_{p}$ since $H_{1}\left(a_{0}\right) \in p R_{p}$ and the elements $\bar{a}_{0}, \bar{a}_{p-1}$ are $p$-dependent. Thus by $(8,1), D_{1}(\pi) \in \pi^{3} R_{p}$. Also $D_{j}\left(a_{s}\right) \in f^{\prime}(\pi) \pi^{3-p-s} R_{p}=\pi^{p+1-s} R_{p}$ for $j \geqq 1, s=0, \ldots, p-1$. Thus, conditions (9) of Lemma 5 are satisfied for $n=1, m=2, q=3$ and again $D \in \mathscr{H}_{u}\left(R_{p}, R_{p}\right)$, $\alpha_{D} \in \mathscr{S}_{1}$ and $\Phi_{1}\left(\alpha_{D}\right)=\delta$.

(3, iii) $\bar{a}_{p-1} \in k^{p}, \bar{a}_{0} \notin k^{p}$. A higher derivation $D$ in $\mathscr{H}_{u}\left(R, R_{p}\right)$ is chosen as in (3, ii). Since $a_{p-1}=b_{p-1}^{p}+p c, H_{1}\left(a_{p-1}\right) \in p R_{p}$. Thus $D_{1}(\pi) \in \pi^{3} R_{p}$ and for the rest the argument of $(3$, ii) applies.

(3, iv) $\bar{a}_{p-1} \notin k^{p}, \bar{a}_{0} \in k^{p}$. We choose $\pi$ so that $a_{0}=1+p b_{0}$. Let $\mathscr{S}$ be a set of 
representatives of a $p$-basis for $k$. We can assume $a_{p-1}$ in $\mathscr{S}$. Let $H_{1}$ in $\mathscr{D}(R)$ induce $\delta$ in $\mathscr{D}(k)$. For $j=2, \ldots, p-1$ and $s \in \mathscr{S}$ we let $H_{j}(s)=0$. For $j=1, \ldots, p-1$ let $D_{j}=\pi^{j} H_{j}$. By $(8,1), D_{1}(\pi) \in \pi^{2} R_{p}\left(D_{1}(\pi) \in \pi^{3} R_{p}\right.$ unless $\left.D_{1}\left(a_{p-1}\right) \notin \pi^{2} R_{p}\right)$. Also, $D,(\pi) \in \pi^{3} R_{p}$ for $j=2, \ldots, p-1$. The terms $A_{p}+B_{p}$ of $(8, \mathrm{p})$ have $\left[D_{1}(\pi)\right]^{p}$ as the unique summand of minimum valuation, if $D_{1}(\pi) \notin \pi^{3} R_{p}$. In any case, $D_{p}$ is defined by $D_{p}(s)=0$ for $s \in \mathscr{S}, s \neq a_{p-1}$ and $D_{p}\left(a_{p-1}\right) \in \pi^{2} R_{p}$ is chosen so that $D_{p}(\pi)$ is in $\pi^{3} R_{p}$. Finally $D,(s)=0$ for $s \in \mathscr{S}$ and $j>p$. Again by Theorem A these conditions determine $D$ in $\mathscr{H}_{u}\left(R, R_{p}\right)$. By Lemma $1 D_{j}(R) \subset \pi^{3} R_{p}$ for $j>p$. Again we invoke Lemma 5 with $n=2, m=p+1$ and $q=3$ to show that $D \in \mathscr{H}_{u}\left(R_{p}, R_{p}\right), \alpha_{D} \in \mathscr{S}_{1}$ and $\Phi_{1}\left(\alpha_{D}\right)=\delta$.

$(3, \mathrm{v}) \bar{a}_{0} \in k^{p}, \bar{a}_{p-1} \in k^{p}$. Again it may be assumed that $a_{0}=1+p b_{0}$. We choose any $H \in \mathscr{H}(R, R)$ such that $H_{1}$ induces a given $\delta \in \mathscr{D}(k)$ and let $D_{j}=\pi^{j} H_{j}, j \geqq 1$. Lemma 5 applies with $n=1, m=2$ and $q=3$.

Case 4. $v\left(f^{\prime}(\pi)\right)=2 p-1, \bar{a}_{0} \notin k^{p}, i>1$. Let $H \in \mathscr{H}(R, R)$ be chosen so that $H_{j}\left(a_{0}\right) \in p R_{p}$ for $j=1, \ldots, p-1$ and $H_{1}$ induces a given $\delta \in \mathscr{D}(k)$ for which $\delta\left(\bar{a}_{0}\right)=0$. Let $D=\left\{\pi^{i j} H_{j}\right\}$. Since by $(8,1) D_{1}(\pi) \in \pi^{i+1} R_{p}$ and, by inspection, $D_{j}\left(a_{s}\right)$ $\in f^{\prime}(\pi) \pi^{i+1-p}, s=0, \ldots, p-1, j \geqq 1$, we see by Lemma 5 that $\sum D_{j}(\pi) \in \pi^{i+1} R_{p}$. Thus $\alpha_{D} \in \mathscr{S}_{i}$ and $\Phi_{i}\left(\alpha_{D}\right)=\delta$. Clearly, $D \in \mathscr{H}_{u}\left(R_{p}, R_{p}\right)$.

Case 5. $v\left(f^{\prime}(\pi)\right)=2 p-1, \bar{a}_{0} \in k^{p}, i>1$. We can assume that $a_{0}=1+p b_{0}$. Let $H \in \mathscr{H}(R, R)$ be such that $H_{1}$ induces a given $\delta \in \mathscr{D}_{k}$. Let $D=\left\{\pi^{i j} H_{j}\right\}$ and argue as above.

Case 6. $v\left(f^{\prime}(\pi)\right)=2 p-1, \bar{a}_{0} \notin k^{p}, i=1$. Let $\delta \in \mathscr{D}(k), \delta\left(\bar{a}_{0}\right)=0$, and let $H_{1}$ in $\mathscr{D}(R)$ induce $\delta$. Let $\mathscr{S}$ be a set of representatives in $R$ of a $p$-basis for $k$ with $a_{0}$ in $\mathscr{S}$. We define $K_{1} \in \mathscr{D}(R)$ as follows: $K_{1}\left(a_{0}\right)=\pi^{-p}\left(H_{1}\left(a_{0}\right)\right)$ and $K_{1}(s)=0$ for $s \in \mathscr{S}, s \neq a_{0}$. By Theorem A, these conditions determine a derivation on $R$. The derivation $D_{1}=\pi H_{1}-\pi^{p+1} K_{1}$ has the property $D_{1}\left(a_{0}\right)=0$ and is the first map of $D \in \mathscr{H}_{u}\left(R, R_{p}\right)$. For the rest, we define $D_{j}(s)=0$ for $s \in \mathscr{S}$ and $j>1$. By Theorem A, $D \in \mathscr{H}_{u}\left(R, R_{p}\right)$. By Lemma $1 D_{1}(R) \subset \pi R_{p}$ and $D_{j}(R) \subset \pi^{2} R_{p}$ for $j \geqq 1$. The conditions of Lemma 5 are fulfilled for $n=1, m=1$ and $q=3$. Moreover, $\phi_{1}\left(\alpha_{D}\right)=\delta$.

Case 7. $v\left(f^{\prime}(\pi)\right)=2 p-1, \bar{a}_{0} \in k^{p}, i=1$. Again, $\pi$ is chosen so that $a_{0}=1+p b_{0}$. We have the situation (3) with $t \geqq p$ and $\bar{v}=\bar{b}_{0}$. Thus, in deference to Lemma 2 , we choose $\delta \in \mathscr{D}(k)$ so that $\delta\left(\bar{b}_{0}\right)=0$ and let $H_{1} \in \mathscr{D}(R)$ induce $\delta$. Let $H \in \mathscr{H}(R, R)$ be any higher derivation on $R$ with the given $H_{1}$ as the first map. Let $D=\left\{D_{j}\right\}$ where $D_{j}=\pi^{j} H_{j}, j \geqq 1$. Let $n=m=1, q=3$ in Lemma 5 and we conclude that $\sum D_{j}(\pi) \in \pi^{3} R_{p}$. Again we have the desired conclusion and Lemma 6 is proved.

The next series of lemmas are concerned with automorphisms in the "gap" between $\mathfrak{S S}_{i}$ and $\mathfrak{S}_{i}$.

LEMMA 7. If $\pi$ is a prime element of $R_{p}$ and $\pi^{p}=-p u$ where $\bar{u} \notin k^{p}$, then, given $i \geqq 2$, there is a $D \in \mathscr{H}_{u}\left(R_{p}, R_{p}\right)$ such that $\alpha_{D} \in \mathscr{S}_{i}$ and $\alpha_{D}(\pi)=\pi+\pi^{i} a$ where $\bar{a}$ is any given element of $k$. Hence $\mathscr{B S}_{i} / \mathfrak{S}_{i}$ is isomorphic to $k^{+}$.

Proof. We assume (6) to be the minimum function of $\pi$ over $R$ and thus $\bar{a}_{0} \notin k^{p}$. 
Let $\mathscr{S}$ be a set of representatives in $R$ of a $p$-basis for $k$ with $a_{0} \in \mathscr{S}$. With $a$ chosen arbitrarily in $R_{p}$ we define a derivation $D_{1}$ mapping $R$ into $R_{p}$ by $D_{1}\left(a_{0}\right)=$ $-p^{-1} f^{\prime}(\pi) \pi^{i} a$ and $D_{1}(s)=0$ for $s \in \mathscr{S}, s \neq a_{0}$. Then $D_{1}(R) \subset f^{\prime}(\pi) \pi^{i-p} R_{p}$ by Lemma 1 and by $(8.1), D_{1}(\pi) \equiv \pi^{i} a, \bmod \pi^{i+1} R_{p}$. Let $D_{j}(s)=0, s \in \mathscr{S}, j=2, \ldots, p-1$. If $i=2$, the term $\left[D_{1}(\pi)\right]^{p}$ in $A_{p}$ of $(8, \mathrm{p})$ makes it necessary to consider cases.

Case 1. $i>2$ or $v\left(f^{\prime}(\pi)\right)<2 p-2$. In this case we let $D_{j}(s)=0, s \in \mathscr{S}, j>p-1$. Thus, by Lemma $1, D_{j}(R) \subset\left[f^{\prime}(\pi) \pi^{i-p}\right]^{j} R_{p}, j \geqq 1$, and if $j>1, D_{j}(R) \subset f^{\prime}(\pi) \pi^{i+1-p}$ since $f^{\prime}(\pi) \in \pi^{p} R_{p}$. The conditions of Lemma 5 are fulfilled for $n=2, m=p+1$ and $q=i+1$. Thus $D$ extends to $R_{p}$, is uniformly convergent on $R_{p}$ and $\sum_{j=2}^{\infty} D_{j}(\pi)$ $\in \pi^{i+1} R_{p}$. In particular then, $\sum_{j=1}^{\infty} D_{j}(\pi) \equiv \pi^{i} a, \bmod \pi^{i+1} R_{p}$.

Case 2. $i=2, v\left(f^{\prime}(\pi)\right) \geqq 2 p-2$. In this case we choose $D_{p}(s)=0, s \in \mathscr{S}, s \neq a_{0}$ and $D_{p}\left(a_{0}\right) \in \pi^{p} R_{p}$ so that $D_{p}(\pi)$ will be in $\pi^{3} R_{p}$. Again, we let $D_{j}(s)=0$ for $j>p$, $s \in \mathscr{S}$ and apply Lemma 5 with $n=2, m=p+1$ and $q=3$, obtaining the same conclusion as in Case 1.

The map $\tau_{i}: \mathscr{G}_{i} \rightarrow k^{+}$given by $\tau_{i}(\alpha)=\bar{a}$ where $\alpha(\pi)=\pi+\pi^{i} a$, is a homomorphism with kernel $\mathfrak{S}_{\mathfrak{i}}$ and evidently maps onto $k^{+}$if $i \geqq 2$.

LEMMA 8. If $\pi$ is a prime element of $R_{p}, \pi^{p}=-p u$, and $\bar{u} \in k^{p}$ then $\mathscr{S S}_{i}=\mathfrak{S}_{i}$ for $i>1$ unless $i=2$ and $t$ of (3) is $p-1$. If $t=p-1$ the following are equivalent.

(a) $\bar{v}$ has $a(p-1)$ th root in $k$.

(b) $R_{p}$ is Galois over $R$.

(c) $\mathfrak{S S}_{2} \neq \mathfrak{S}_{2}$.

(d) $\mathfrak{S}_{2} / \mathfrak{S}_{2}$ is the group of order $p$.

Proof. Let $\alpha$ be in $\mathfrak{S}_{i}$. Then $\alpha=\varepsilon+\pi^{i} \alpha^{*}$. The relation

$$
[\alpha(\pi)]^{p}-\pi^{p}=p\left[1+[\alpha(\pi)]^{t} \alpha(v)\right]-p\left(1+\pi^{t} v\right)
$$

becomes

$$
\begin{aligned}
p \pi^{i+p-1} \alpha^{*}(\pi)+\cdots+ & \pi^{i p}\left[\alpha^{*}(\pi)\right]^{p} \\
= & p\left[\pi^{t}+t \pi^{t-1+i} \alpha^{*}(\pi)+\cdots+\pi^{t i}\left(\alpha^{*}(\pi)\right)^{t}\right] \pi^{i} \alpha^{*}(v) \\
& +p\left[t \pi^{t-1+i} \alpha^{*}(\pi)+\cdots+\pi^{t i}\left(\alpha^{*}(\pi)\right)^{t}\right] v
\end{aligned}
$$

If $i>2$ the unique term having minimal valuation on the left side of (15) is $p \pi^{i+p-1} \alpha^{*}(\pi)$. If $p \nmid t$ the unique term of minimal valuation on the right is $p t \pi^{t-1+i} v \alpha^{*}(\pi)$, unless $\alpha^{*}(\pi)$ is in $\pi R_{p}$. Thus, either $\alpha^{*}(\pi) \in \pi R_{p}$ or $t+i-1=p+i-1$, which cannot be. Thus, if $i>2$ and $p \nmid t$, then $\alpha \in \mathfrak{S}_{i}$ or $\mathscr{S S}_{i}=\mathfrak{S}_{i}$. If $p \mid t$ and $i \geqq 2$ the left side of (15) has valuation less than the right side unless $\alpha^{*}(\pi) \in \pi R_{p}$. Thus again $\mathfrak{S}_{i}=\mathfrak{S}_{i}$.

If $i=2$ and $p \nmid t$, the unique term of minimal valuation on the left side of (15) is $\pi^{2 p}\left[\alpha^{*}(\pi)\right]^{p}$, assuming $\alpha^{*}(\pi)$ to be a unit. The corresponding term on the right is $p t \pi^{t+1} \alpha^{*}(\pi) v$. Thus, $2 p=p+t+1$ or $t=p-1$. So, if $t \neq p-1$, $\mathbb{S}_{2}=\mathfrak{S}_{2}$. If $t=p-1$, then by (15), $\pi^{2 p}\left[\alpha^{*}(\pi)\right]^{p} \equiv p(p-1) \pi^{p} \alpha^{*}(\pi) v, \bmod \pi^{2 p+1} R_{p}$, or, using (3), $\left[\alpha^{*}(\pi)\right]^{p-1}$ $\equiv(p-1) v, \bmod \pi R_{p}$. Thus, $(p-1) \bar{v}$, or $\bar{v}$, is a $(p-1)$ th root in $k$ and the residue 
of $\alpha^{*}(\pi)$ is a $(p-1)$ th root of $(p-1) \bar{v}$. We have shown that (c) $\rightarrow$ (d) $\rightarrow$ (a). A theorem of Wishart [4, Theorem 4.15] asserts that (a) $\rightarrow$ (b).

Suppose, finally, that $\alpha$ in $\mathfrak{S}_{1}$ leaves $R$ element-wise fixed. Then, if $\alpha(\pi)=\pi+\pi^{i} b$, $\alpha \in \mathscr{S}_{i}$. Thus, if $\alpha \neq \varepsilon$, then $\alpha \in \mathscr{S S}_{r}, \alpha \notin \mathfrak{S}_{r}$ for some $r>1$. Evidently, $r=2$ and (b) $\rightarrow$ (c). This fact was also observed by Wishart [4, Corollary 4.16] who noted that if $\bar{u} \in k^{p}$ then $R_{p}$ is Galois over $R$ if and only if $\mathscr{S S}_{2} \neq \mathfrak{S}_{2}$. It follows from Lemma 7 that if $\bar{u} \notin k^{p}$, then $\mathfrak{S}_{2}$ can be different from $\mathfrak{S}_{2}$ without $R_{p}$ being Galois over $R$.

LEMMA 9. If $\mathfrak{S}_{2} \neq \mathfrak{S}_{2}$, then, for each $\alpha \in \mathfrak{S S}_{2}$, there is a $D$ in $\mathscr{H}_{u}\left(R_{p}, R_{p}\right)$ such that $\alpha \alpha_{D}^{-1} \in \mathfrak{S}_{2}$ if and only if, in (3), $\bar{v} \notin k^{p}$.

Proof. Assuming first that $\bar{v} \notin k^{p}$ it follows from Lemma 8 that in (3), $t=p-1$ and $\bar{v}$ is a $(p-1)$ th root in $k$. Assuming (6) to be the minimal polynomial of $\pi$ over $R$, relation (3) with $t=p-1$ implies that $a_{1}, \ldots, a_{p-2}$ are in $p R, \bar{a}_{p-1}(=-\bar{v})$ is a $(p-1)$ th root in $k, v\left(f^{\prime}(\pi)\right)=2 p-2$ and $a_{0}=1+p b_{0}$.

Let $w$ be a unit in $R_{p}$ such that $\bar{w}$ is a $(p-1)$ th root of $\bar{a}_{p-1}(p-1)$. We wish to construct $D \in \mathscr{H}_{u}\left(R_{p}, R_{p}\right)$ such that $\alpha_{D} \in \mathscr{F}_{2}$ and $\alpha_{D}(\pi) \equiv \pi^{2} w, \bmod \pi^{3} R_{p}$.

Let $\mathscr{S}$ be a set of representatives in $R$ for a $p$-basis of $k$ chosen to include $a_{p-1}$. Then $D_{1}$ is defined by $D_{1}\left(a_{p-1}\right)=-f^{\prime}(\pi) \pi^{2} w / p \pi^{p-1}, D_{1}(s)=0$ for $s \in \mathscr{S}, s \neq a_{p-1}$. By Lemma $1 D_{1}(R) \subset \pi^{2} R_{p}$ and by $(8.1) D_{1}(\pi) \equiv \pi^{2} w, \bmod \pi^{3} R_{p}$. For $j=2, \ldots, p-1$ and $s \in \mathscr{S}, D_{j}(s)=0$. By $(8,2)$ to $(8, p-1), D_{j}(\pi) \in \pi^{3} R_{p}$ for $j=2, \ldots, p-1$. The term $\left[D_{1}(\pi)\right]^{p}$ in $(8, \mathrm{p})$ leads us to define $D_{p}$ by $D_{p}\left(a_{p-1}\right)=-\pi^{2 p} w^{p} / p\left(\pi^{p-1}\right)$ and $D_{p}(s)=0, s \in \mathscr{S}, s \neq a_{p-1}$. Since each term of $(8, \mathrm{p})$ in $A_{p}+B_{p}$ is in $\pi^{2 p+1} R_{p}$ save $\left[D_{1}(\pi)\right]^{p}$ and $\left[D_{1}(\pi)\right]^{p} \equiv \pi^{2 p} w^{p}, \bmod \pi^{2 p+1} R_{p}$, we have $D_{p}(\pi) \in \pi^{3} R_{p}$. Finally, we let $D_{j}(s)=0$ for $s \in \mathscr{S}$ and $j>p$. Then $D_{j}(R) \subset \pi^{2} R_{p}$ for $j>p$ and by Lemma 5 with $n=2, m=p+1$ and $q=3$, we conclude that $\sum D_{j}(\pi)$ converges and $\sum_{j=2}^{\infty} D_{j}(\pi) \in \pi^{3} R_{p}$.

It remains to show that $\alpha_{D}$ is in $\mathscr{S S}_{2}$. We have shown that $\alpha_{D}(\pi)-\pi$ is in $\pi^{2} R_{p}$ and it is shown below that

$$
\alpha_{D}(s)-s \in \pi^{2} R_{p}, \quad s \in \mathscr{S} .
$$

If $s \in \mathscr{S}, s \neq a_{p-1}$ then $\alpha_{D}(s)=s$ by definition of $D$. Since $D_{j}\left(a_{p-1}\right)=0$ for $j \neq 1, p$ it is sufficient to show that $D_{1}\left(a_{p-1}\right)+D_{p}\left(a_{p-1}\right) \in \pi^{2} R_{p}$. Now, $f^{\prime}(\pi) \equiv(p-1) a_{p-1} \pi^{p-2}$, $\bmod \pi^{p-1} R_{p}$. Also $w^{p-1} \equiv(p-1) a_{p-1}, \bmod \pi R_{p}$, by choice of $w$. Using these facts as well as the congruence $\pi^{p} \equiv-p, \bmod \pi^{p+1} R_{p}$, leads to the conclusion $D_{1}\left(a_{p-1}\right)$ $+D_{p}\left(a_{p-1}\right)=-f^{\prime}(\pi) \pi^{2} w / p \pi^{p-1}-\pi^{2 p} w^{p} / p \pi^{p-1} \in \pi^{2} R_{p}$

Since $\alpha_{D}$ is inertial, $\alpha_{D}\left(a^{p}\right)-a^{p} \in \pi^{p} R_{p}$ and every unit in $R$ is, $\bmod p R$, a polynomial in elements of $\mathscr{S}$ with coefficients in $R^{p}$. It follows that $\alpha_{D}(a)-a \in \pi^{2} R_{p}$ for $a$ in $R$. Thus $\alpha$ is in $\mathbb{S F}_{2}$.

It was shown in the proof of Lemma 8 that if $\alpha \in \mathbb{F}_{2}$ then $\alpha=\varepsilon+\pi^{2} \alpha^{*}$ and $\alpha \in \mathfrak{H}_{2}$ or the residue of $\alpha^{*}(\pi)$ is a $(p-1)$ th $\operatorname{root}$ of $(p-1) \bar{v}=\bar{a}_{p-1}(p-1)$. Thus if we choose $w$, in the construction of $D$, to be $\alpha^{*}(\pi)$, then $\alpha \alpha_{D}^{-1} \in \mathfrak{H}_{2}$.

If $\bar{v} \in k^{p}$ then $v=v_{0}^{p}+\pi v_{1}$. Thus $\bar{a}_{p-1}=\bar{v}_{0}^{p}$ and $\bar{b}_{0}=\bar{v}_{1}$ where, again, $a_{0}=1+p b_{0}$. We choose $c_{0}$ and $c_{1}$ in $R$ so that $a_{p-1}=c_{0}^{p}+p c_{1}$. Let $D \in \mathscr{H}_{c}\left(R_{p}, R_{p}\right)$ be such that 
$\alpha_{D} \in \mathscr{S}_{2}, \alpha_{D} \notin \mathfrak{S}_{2}$. There is, then, a first index $j$ such that $f^{D_{j}}(\pi) \in f^{\prime}(\pi) \pi^{2} R_{p}$ and

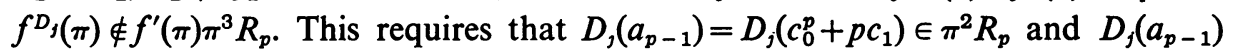
$\notin \pi^{3} R_{p}$. However, $D_{j}(R) \subset \pi R_{p}$ and hence $D_{j}\left(c_{0}^{p}+p c_{1}\right) \in \pi^{p} R_{p}$. We have a contradiction. Thus $\mathscr{B}_{2} \cap \mathbb{S}_{D} \subset \mathfrak{S}_{2}$, and Lemma 9 is proved.

For $i>1$ and $\alpha \in \mathfrak{F}_{i}$ there is a $D \in \mathscr{H}_{u}\left(R_{p}, R_{p}\right)$ such that $D\left(R_{p}\right) \subset \pi^{i} R_{p}$ (see (12)) and $\alpha \alpha_{D} \in \mathscr{S}_{i+1}$. Also if $i>2$ and $\alpha \in \mathscr{S}_{i}$ then $\alpha \in \mathscr{S}_{i}$ or there is a $D \in \mathscr{H}_{u}\left(R_{p}, R_{p}\right)$ such that $D\left(R_{p}\right) \subset \pi^{i} R_{p}$ and $\alpha \alpha_{D} \in \mathfrak{S}_{i}$. This follows from Lemma 7, Case 1 of the proof of Lemma 7 and Lemma 8. Thus, given $\alpha \in \mathfrak{S}_{2}$ there is a sequence $\left\{D^{(n)}\right\}$, $D^{(n)} \in \mathscr{H}_{u}\left(R_{p}, R_{p}\right)$ such that, $D^{(n)}\left(R_{e}\right) \subset \pi^{s_{n}} R_{p}$ where $\lim _{n} s_{n}=\infty$, and $\ddot{\alpha}=\alpha_{D_{1}} \alpha_{D_{2}} \ldots$ $\alpha_{D_{2 n}}, \bmod \mathfrak{S}_{n+2}$. By Lemma 3 , there is a $D \in \mathscr{H}_{u}\left(R_{p}, R_{p}\right)$ such that $\alpha=\alpha_{D}$.

By Lemma 6 and Lemma 9 , we conclude that $\mathscr{S}_{D}$ and $\mathscr{S}\left(R_{p}, R\right)$ together generate ङ5. If $\beta$ is an automorphism on $R$ and $D \in \mathscr{H}_{c}\left(R_{e}, R_{e}\right)$ then $H=\left\{H_{i}\right\}$ where $H_{i}=\beta^{-1} D_{\mathrm{t}} \beta$ is also in $\mathscr{H}_{c}\left(R_{e}, R_{e}\right)$. If $D$ converges uniformly so does $H$. Thus $\mathscr{S}_{D}$ is an invariant subgroup of $G$ the automorphism group of $R_{p}$. Hence $\mathscr{S}_{1}=$ $\mathscr{G}_{D} \cdot \mathbb{S}\left(R_{p}, R\right)$. These observations along with Lemmas 8 and 9 prove Theorem 1. Theorem 2 follows directly from Lemmas $2,4,6,7$ and 8 .

\section{REFERENCES}

1. N. Heerema, Derivations on p-adic fields, Trans. Amer. Math. Soc. 102 (1962), 346-351.

2. - Convergent higher derivations on local rings, Trans Amer. Math. Soc. 132 (1968), $31-44$.

3. J. Neggers, Derivations on $\bar{p}$-adic fields, Trans. Amer. Math. Soc. 115 (1965), 496-504.

4. E. Wishart, Higher derivations on $\bar{p}$-adic fields, Dissertation, Florida State Univ., Tallahassee, 1965.

Florida State University,

Tallahassee, Florida 